\title{
HIV infection among ethnobotanicals IV drug users followed in "Dr. Victor Babes" Clinical Hospital for Infectious and Tropical Diseases
}

\author{
Ionut Popa' ${ }^{1}$, Simona Erscoiul, ${ }^{1,2}$ Irina lanache ${ }^{1}$, Olivia Burcos ${ }^{1}$, \\ Roxana Radoi ${ }^{1}$, Cristiana Oprea ${ }^{1,2}$ \\ 1"Dr. Victor Babes" Clinical Hospital for Infectious and Tropical Diseases, Bucharest, Romania \\ 2"Carol Davila" University for Medicine and Pharmacy, Bucharest, Romania
}

\begin{abstract}
$\mathrm{HIV/HCV}$ co-infection is permanently increasing in our country especially among intravenous drug users (IDUs) from low socio-economical risk categories. HIV/HCV co-infection among IDUs is associated with a complex pathology, including psychiatric disorders due to severe addiction syndrome, bacterial infection at the injection site and systemic bacterial infections of various degrees of severity. Due to HIV related immunosuppression, pulmonary or extra-pulmonary tuberculosis is frequently diagnosed, with major risk for dissemination Mycobacterium tuberculosis in the general population, including MDR or XDR TB strains.
\end{abstract}

Keywords: HIV-AIDS infection, HCV chronic hepatitis, ethnobotanical drugs, tuberculosis

In the actual social context with a large amount of young people using injectable drugs (either heroine or newly "ethnobotanical" drugs) in our country we noticed permanently increased incidence of HIV-AIDS infections and HCV hepatitis (1). Reduced access to medical care for this high risk category of patients, due to their poor economic and educational status, (including homelessness, lack of health insurance, no health insurance card) but also due to low adherence to antiretroviral therapy impact negatively the progression of $\mathrm{HIV} / \mathrm{HCV}$ coinfection. Intravenous drug users are often seen in hospital only in the advanced stage of illness, being diagnosed with HIV as "late presenters", with CD4 cell count below 350 cells/ml and with severe opportunistic infections or other AIDS defining conditions(2) The most frequent opportunistic infection among IDUs admitted in our clinic is due to Mycobacterium tuberculosis, resulting in high risk for transmission in the general population (3).
"Ethnobotanical" drugs represent a heterogeneous category of vegetal substances impregnated with several amphetamine derivates and/or cannabinoids, resulting in a large diversity of products. Their commercialisation was firstly permitted in our country in so called "dream shops", therefore being named by users "legal drugs" $(4,5)$. Even if they were original designed as inhalation drugs, IDUs discovered quickly that intravenous injection enhance the hallucinogens effect. Having a low price (10-30 Lei/box), ethnobotanicals usage become largely affordable in the general population, particularly for low status people with poor access to medical care for reasons mentioned above. Furthermore, sharing needles is common in this group of patients, being responsible for over $97 \%$ of IVDU infected with HCV and more than 50\% HIV positive IDUs hospitalised today in Romania (6). (with many more yet unknown cases). Even if this shops were closed in 2013, this only slightly de- 
creased the number of newly $\mathrm{HIV} / \mathrm{HCV}$ transmission, as products are still largely available on the black market and on the internet).

Between $1^{\text {st }}$ January 2008 and 31 December 2016, 1224 newly HIV infected IDUs were registered in Romania, according to the report of the "National Centre for Fight Against HIV-AIDS" from Matei Bals National Institute for Infectious Diseases (1). The large majority of these persons live in the area around Bucharest, 790 of them (70.2\%) being followed in our hospital care (Fig. 1).

The management of IDUs is complex, difficult and expensive for multiple reasons.

Many patients came to our hospital without health insurance, needing emergency hospitalisation, often being collected by the ambulance directly from the streets, in many cases without any ID papers, because of that their medical care may not be supported by the National Insurance House. Furthermore, many patients are homeless, with no income and no family support, and even if their medical emergency is successfully managed, when they are discharged they go back on street resuming their habits, therefore sooner or later come back to our hospital.

IDUs frequently have psychiatric disorders because of the addiction syndrome, with aggressive behaviour towards medical personnel and also towards other patients, demanding permanent psychiatric medication and management. This is difficult to achieve in an infectious diseases hospital. They may also have neurocognitive disorders be- cause of HIV and prolonged intravenous drug usage. After a slight improvement in their clinical status they often ask for self-discharge, often to came back to the hospital several hours (days) after with altered health status.

Intravenous drug usage the streets on street happens for sure in improper conditions. Beside commonly usage of needles and syringes mentioned above, we have to consider that they are using septic fluid as solvents (like kitchen water, water from the street, sodas), which favours different bacterial infections, with various severity depending on localisation and aetiology. IDUs develop often cellulitis and/or ulcerations, septic phlebitis on the administration site due to para venous self-injections. This affect especially upper and lower limbs, but sometimes inguinal and cervical region may be involved when they do not obtain proper femoral or jugular puncture.

Systemic bacterial involvement is also possible. Bacterial pneumonia is frequent among IDUs patients, often complicated with lung abscess, emphysema and/or pneumothorax, in case of staphylococcal involvement. Bacterial endocarditis is also found, involving mainly the right tricuspidian valve, with milder symptomatology compared to the left mitral endocarditis, but high risk of silent progression in the absence of treatment, due to pulmonary hypertension and consequent cardiac failure. Cerebral bacterial involvement may also happen, often difficult to distinguish from the underlying psychiatric disorder. However brain ab-

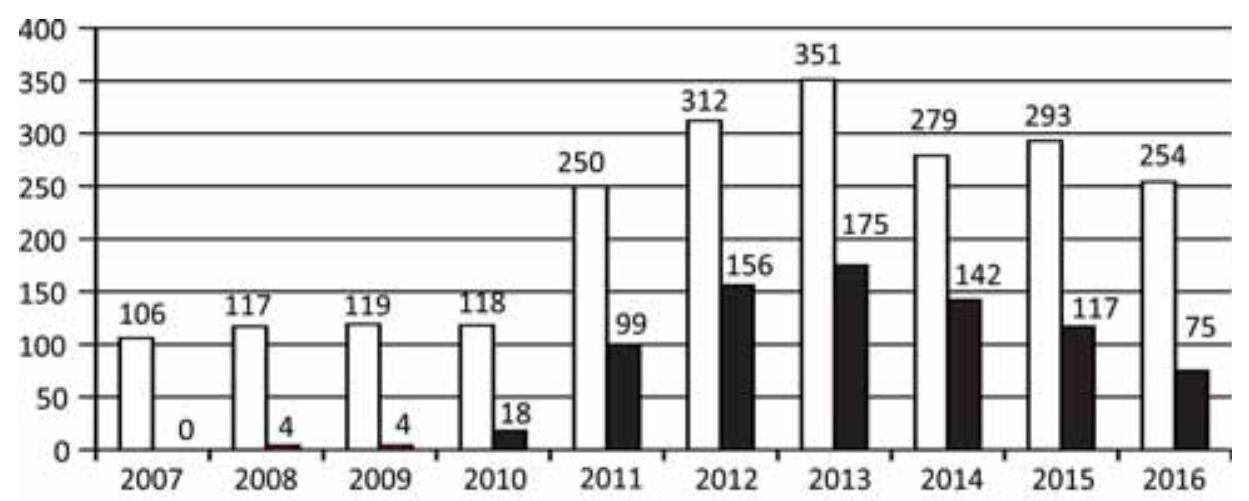

\footnotetext{
$\square$ New cases with HIV infections

Drug users with HIV infection
}

FIGURE 1. Proportion of IDUs among newly HIV infected patients in "Victor Babes" Hospital, between 2007 and 2016 
scess and bacterial meningitis may came as surprise diagnosis in IDUs. In fact, we are often confronting systemic self-progressive bacterial infections with multiples sites involved, in many cases sepsis criteria are accomplished, with high mortality risk. Staphylococcus aureus is the most frequent etiological agent among IDUs, initially with methicillin sensitivity, but since these patients have multiple hospitalisations the risk to encounter methicillin resistance strains and/or to acquire other gram-negative bacteria with high spectrum resistance to antibiotics is particularly high.

Lack of proper initiation on antiretroviral treatment (often because of poor adherence) contribute to progressive immunosuppression. Due to the epidemiological conditions existing in our country, the first major opportunistic infection is pulmonary tuberculosis (TB). In many cases with poor inflammatory pulmonary response associated with high immunosuppression, pulmonary tuberculosis is often missed from clinical and radiological diagnosis, rapid haematogenous dissemination of Mycobacterium tuberculosis occurs and we may diagnose "de novo" lymph node tuberculosis, cerebral/meningeal tuberculosis, urinary and intestinal tuberculosis, and even severe forms of septic tuberculosis. In our clinic we confirmed TB involvement in more than $1 / 3$ from all IDUs either by classical bacterial exam (smear and/or cultures), either by modern methods like PCR for Mycobacterium tuberculosis or GeneXpert technique. The risk for contagion for health care workers is very high, also for other patients and even for the general population, since not all these patients may be restricted in hospital or TB medical facilities. What concerns us further is the appearance of resistant TB strains, type MDR and even XDR tuberculosis among IVDU, demanding expensive and prolonged treatment. Clinically patients may have persistent febrile syndrome despite classical TB treatment, with high risk for unfavourable outcome and mortality. In this cases rapid rifampin-resistant strain identification, possible now by GeneXpert technique is mandatory and lifesaving.

Beside TB infection, other opportunistic infections and malignancies may affect an immunological compromised human host. Even if they were rare at the beginning of the epidemics of HIV infection among IDUs (because patients were recently infected with HIV and had a reasonable immunity), in the absence of antiretroviral treatment, the whole spectrum of immunological infections AIDS defining become noticeable among IDUs patients. We have to consider now differential diagnosis from pulmonary TB with Pneumocystis jirovecii pneumonia or with CMV pneumonia. Also brain abscess due to cerebral toxoplasmosis may be distinguished from brain TB and/or Staphylococcus aureus brain abscess. Cryptococcus neoformans meningitis have to be distinguished for TB meningitis. HIV encephalitis and multiple progressive leukoencephalopathy have to be differentiated from psychiatric disorders. Also AIDS defining malignancies, Kaposi sarcoma, primary cerebral lymphoma and nonHodgkin lymphoma started to be noticed among IDUs in our clinic.

Child-bearing women may also use injectable drugs, and since they do not came to regular clinical exams they are not tested on time for HIV, hepatic viruses or other infections which may affect the new born child. In fact they came very late to hospital, often only when they are in labour and so they are diagnosed with HIV after giving birth. In the absence of antiretroviral treatment, the risk of mother to child HIV transmission is very high, and because of that the IDUs mothers were the only cases noticed in our clinic for mother-to-child HIV transmission in the last years. They also have (a smaller) risk for HCV transmission.

Even if it is yet mostly unconsidered by many clinicians, HCV infection progression toward liver cirrhosis and hepatocellular carcinoma in IDUs is a worrying reality. Immunologically-suppression HIV related fasten progression of $\mathrm{HCV}$ infection compared to other categories of HCV patients. Furthermore, response rate to classical interferon/ribavirin treatment on HIV positive patients is lower and treatment tolerability is lower. For this reasons, in some countries HIV/HCV coinfection is considered priority for interferon-free treatment, since these patients represent an important reservoir and a high risk for dissemination HCV in the general population. But if the patient continue to use injectable drugs after being cured, re-infection may appear at any time.

In conclusion injectable drug usage is a dramatic reality in our country, and by affecting mainly young people from big urban areas (active popula- 
tion), a major contributor in $\mathrm{HIV} / \mathrm{HCV}$ dissemination into the general population. Medical and social care towards IDUs in hospitals and "harm reduction" facilities, including needle-exchange programs and methadone substitution therapy is still insufficient, both for reducing contagion of HIV/ $\mathrm{HCV}$ infection among injectable drug users and also to prevent progression toward AIDS and death on those infected. TB infection is frequent among IDUs nowadays having a major risk for dissemination of resistant TB strains into the general population.

In order to improve the medical care for patients from vulnerable risk groups factors, a large number of IDUs have been included in the project HepCare
Europe - 709844, co-founded by the European Commission. The main objective of HepCare project is to enhance rapid HCV diagnosis by "point of care testing" and so to make possible proper $\mathrm{HCV}$ management for these patients. The project is also aiming to elaborate educational programs for family doctors and for patients regarding HCV diagnosis tools, prevention of $\mathrm{HCV}$, how to obtain social support and finally to improve access to direct antiviral agents treatment (DAA).

This paper has been elaborated as part of Hepcare Europe Project - 709844, co-financed by Health Programme CHAFEA by the European Commission

Conflict of interest: none declared

\section{REFERENCES}

1. CNLAS, Institutul Naţional de Boli Infecţioase „Matei Balş“, www. cnlas.ro, 31 decembrie 2016

2. lanache I., Calistru P., Tardei G., Ruta S., Oprea C. Late presentation in HIV-infected injecting drug users - a huge challenge for the Romanian health-care system, Rom J Leg Med (24) 122-127 (2016)

3. Oprea C., Rădoi R., Erşcoiu S., Nica M., Tardei G., Ruta S., Calistru P., Ceauşu Em. Tripla infecţie HIV, hepatita C şi tuberculoză - 0 adevarată epidemie la utilizatorii de droguri injectabile - Revista Română de Boli Infecțioase vol XVII, nr. 2, 2014

4. Maftei L. The main characteristics of the Romanian illegal drug markets; Eastern Journal of European Studies (EJES), Volume 3, Issue1, June 2012, p. 189-204

5. European Monitoring Centre for Drugs and Drug Addiction (EMCDDA) and National anti-Drug Agency (NAA); National Report to the European Monitoring Centre for National (EMCDDA), Romania 2014 (Raport Naţional privind Situaţia Drogurilor 2014, România; Noi evoluţii şi tendinţe)

6. Oprea C., Ceauşu Em., Ruta S. Ongoing outbreak of multiple blood-borne infections in injecting drug users in Romania, Public Health 127 (2013), 1048-1050 